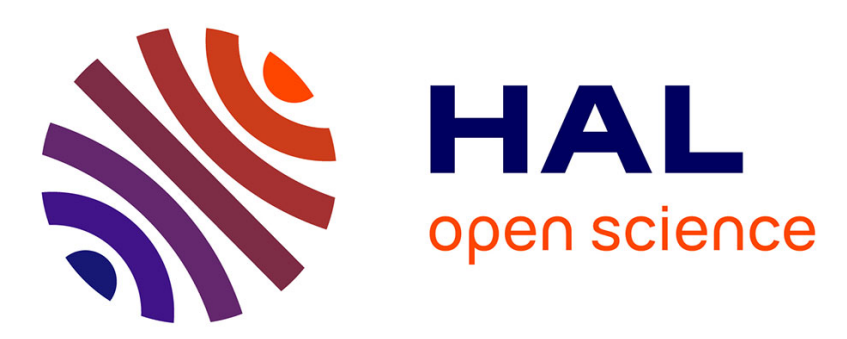

\title{
LMI-based design of dynamic event-triggering mechanism for linear systems
}

Sophie Tarbouriech, Antoine Girard

\section{To cite this version:}

Sophie Tarbouriech, Antoine Girard. LMI-based design of dynamic event-triggering mechanism for linear systems. IEEE Conference on Decision and Control (CDC 2018), Dec 2018, Miami Beach, United States. pp.121-126, 10.1109/CDC.2018.8619103 . hal-02268566

\section{HAL Id: hal-02268566 https://hal.laas.fr/hal-02268566}

Submitted on 22 Jul 2020

HAL is a multi-disciplinary open access archive for the deposit and dissemination of scientific research documents, whether they are published or not. The documents may come from teaching and research institutions in France or abroad, or from public or private research centers.
L'archive ouverte pluridisciplinaire $\mathbf{H A L}$, est destinée au dépôt et à la diffusion de documents scientifiques de niveau recherche, publiés ou non, émanant des établissements d'enseignement et de recherche français ou étrangers, des laboratoires publics ou privés. 


\title{
LMI-based design of dynamic event-triggering mechanism for linear systems
}

\author{
Sophie Tarbouriech and Antoine Girard
}

\begin{abstract}
This paper deals with the design of an eventtriggering mechanism using local information for a linear system controlled by an observer-based feedback controller. The event-triggered control strategy is based on a new dynamic triggering mechanism, which is introduced through an internal dynamic variable. Sufficient conditions based on linear matrix inequalities are proposed to ensure the asymptotic stability of the closed loop together with the avoidance of Zeno behavior. A convex optimization problem leans on these conditions to determine the parameters of the event-trigger rule aiming at reducing the number of control updates. Discussion with respect to the simpler state feedback case is drawn.
\end{abstract}

\section{INTRODUCTION}

The event-triggered control consists in devising eventtriggering mechanisms leading only seldom control updates. In the context of event-triggered control, two objectives can be pursued: (1) Emulation: the controller is a priori predesigned and only the event-triggered rules have to be designed (see, for example, [11], [20], [14], [2], [8] and the references therein), or (2) Co-design: the joint design of the control law and the event-triggering conditions has to be performed (see, for example, (see [4], [15], [1], [10] [3], and the references therein). The current paper comes within the scope of the first case, that is, of the emulation context. Indeed, it is important to have in mind that control systems are connected to generic digital communication networks for implementation, transmission, coding or decoding. Then, event-triggered control strategies have been developed mainly to cope with communication, energy consumption and computation constraints (see, for example, [13], [11], [16], [4] and the references therein).

Note that the most of the papers above cited consider static event-triggered rules, differently from the dynamical case as addressed, for example, in [9], [7], [12], [6] and recently extended to the multi-agent systems case in [21]. In these papers, the idea consists in introducing an additional variable, which is indeed an internal dynamic variable allowing to augment the event-triggered rule with dynamics.

In the current paper, inspired by the approach proposed in [9], a new kind of event-triggering mechanism is developed in an emulation context, which can be seen as complementary to the approach developed in [5]. The originality of the approach relies on: 1) The class of dynamics considered for the internal variable, which extends that one proposed in [9]; 2) The event-triggering mechanism uses only local

S. Tarbouriech is with LAAS-CNRS, University of Toulouse, CNRS, Toulouse, France. Email: tarbour@laas.fr. A. Girard is with Laboratoire des Signaux et Systèmes, CNRS, CentraleSupc, Université ParisSud, Université Paris-Saclay, Gif sur Yvette, Paris, France. Email: antoine.girard@12s.centralesupelec.fr. information available for the control purpose; and 3) The resulting event-triggered law is free from Zeno behavior thanks to the use of an explicit tuning parameter, which plays the role of a minimal dwell time. This is also an alternative route to [6], in which a hybrid dynamical system framework is used. Furthermore, one can highlight that the event-triggered rules of [9] in the dynamic case, or in [19] in the static case, can be embedded in that one studied in this paper.

The paper is organized as follows. Section II describes first the system under consideration. Next the new dynamic triggering mechanism is presented in order to enrich the event generator algorithm managing the controller to decide when the control input has to be updated. Then, the control problem we intend to solve is formally stated. In Section III, the first result (Theorem 1) constitutes the backbone of the paper allowing to prove that the asymptotic stability of the closed-loop system with the proposed triggering mechanism is guaranteed. From this generic result, conditions based on linear matrix inequalities (LMIs) are proposed (Theorem 2). One of the salient facts relies on the dwell time explicitly appearing as a tuning parameter. Associated to these conditions, a simple optimization criterion is proposed to deal with the implicit objective of reducing the number of control updates by orienting the choice of the event-triggering mechanism parameters. In Section IV, two examples adapted from the literature illustrate the efficiency of the proposed approach. Finally, some concluding remarks and directions for potential future works are presented in Section V.

Notation. $\mathbb{N}, \mathbb{R}^{n}, \mathbb{R}^{n \times m}$ denote respectively the sets of nonnegative integers, $n$-dimensional vectors and $n \times m$ matrices. For any matrix $A, A^{\top}$ denotes its transpose and $H e\{A\}=A+A^{\top}$. For any square matrix $A, \operatorname{trace}(A)$ denotes its trace. $\operatorname{diag}\left(A_{1} ; A_{2}\right)$ is a diagonal matrix with block diagonal matrices $A_{1}$ and $A_{2}$. For two symmetric matrices of the same dimensions, $A$ and $B, A>B$ means that $A-B$ is symmetric positive definite. $I$ and 0 stand respectively for the identity and the null matrix of appropriate dimensions. For a partitioned matrix, the symbol $\star$ stands for symmetric blocks. I| . || stands for the Euclidean norm. |.| stands for the absolute value.

\section{Problem Statement}

\section{A. System description}

Consider the following continuous-time linear plant

$$
\left\{\begin{aligned}
\dot{x}_{p}(t) & =A_{p} x_{p}(t)+B_{p} u(t), \\
y_{p}(t) & =C_{p} x_{p}(t)
\end{aligned}\right.
$$


where $x_{p}(t) \in \mathbb{R}^{n}, u(t) \in \mathbb{R}^{m}, y_{p}(t) \in \mathbb{R}^{p}$ are the state, the input and the output of the plant, respectively. Matrices $A_{p}$, $B_{p}, C_{p}$ are constant and of appropriate dimensions. Pairs $\left(A_{p}, B_{p}\right)$ and $\left(C_{p}, A_{p}\right)$ are supposed to be controllable and observable, respectively.

We consider an observer-based feedback controller to stabilize the plant (1) and therefore to drive the output to zero. This controller is defined by:

$$
\left\{\begin{array}{l}
\dot{\hat{x}}(t)=A_{p} \hat{x}(t)+B_{p} u(t)-L\left(y_{p}(t)-\hat{y}(t)\right) \\
\hat{y}(t)=C_{p} \hat{x}(t) \\
u(t)=K \hat{x}(t)
\end{array}\right.
$$

where $\hat{x}(t) \in \mathbb{R}^{n}$ and $\hat{y}(t) \in \mathbb{R}^{p}$ are the state and the output of the observer. Furthermore, $L \in \mathbb{R}^{n \times p}$ and $K \in \mathbb{R}^{m \times n}$ are the observer and controller gains, respectively.

By considering the continuous-time system described by (2), the control design is carried out according to the separation principle. In other words, the observer gain $L$ is designed to make $A_{p}+L C_{p}$ Hurwitz, which leads to the state estimation error dynamics given by

$$
\dot{e}(t)=\left(A_{p}+L C_{p}\right) e(t),
$$

where $e(t)=x_{p}(t)-\hat{x}(t) \in \mathbb{R}^{n}$, is globally asymptotically stable, i.e. $\lim _{t \rightarrow \infty} e(t)=0$. Consequently, the estimation output error $e_{y}(t)=C_{p} e(t)$ also asymptotically converges to zero, i.e. $\lim _{t \rightarrow \infty} e_{y}(t)=0$. Furthermore, the dynamics of the observer is given by:

$$
\dot{\hat{x}}(t)=\left(A_{p}+B_{p} K\right) \hat{x}(t)-L e_{y}(t) .
$$

\section{B. Control implementation}

In this note the objective relies on the way to implement the control input $u$, taking into account that $u$ is implemented through a sample-and-hold mechanism. It is not continuously updated or transmitted to the actuators. Indeed, it is updated at certain instants $\left\{t_{k}\right\}_{k \in \mathbb{N}}$, which form a sequence of strictly increasing positive scalars. Control action is held constant between two successive sampling instants $\left(t_{k}\right.$ and $\left.t_{k+1}\right)$ through a zero order holder. Differently from classical digital control approaches, the sampling interval $t_{k+1}-t_{k}$ is not assumed to be constant but can be seen as an additional control action.

Thus, the closed-loop system can be represented by

$$
\left\{\begin{aligned}
\dot{x}_{p}(t) & =A_{p} x_{p}(t)+B_{p} u\left(t_{k}\right), \\
\dot{\hat{x}}(t) & =A_{p} \hat{x}(t)+B_{p} u\left(t_{k}\right)-L e_{y}(t), \\
u\left(t_{k}\right) & =K \hat{x}\left(t_{k}\right), \quad \forall t \in\left[t_{k}, t_{k+1}\right) .
\end{aligned}\right.
$$

By using the same formulation as in [16] to define $\delta(t)$ :

$$
\delta(t)=\hat{x}\left(t_{k}\right)-\hat{x}(t)
$$

the closed loop can be expressed in terms of $\hat{x}$ and $e$, as follows:

$$
\left\{\begin{array}{l}
\dot{\hat{x}}(t)=\left(A_{p}+B_{p} K\right) \hat{x}(t)+B_{p} K \delta(t)-L e_{y}(t), \\
\dot{e}(t)=\left(A_{p}+L C_{p}\right) e(t), \quad \forall t \in\left[t_{k}, t_{k+1}\right) .
\end{array}\right.
$$

Remark 1: It is important to note that $\delta$ depends only on the observer variables and is therefore available at the controller node. The function $\delta$ corresponds to a measure of the difference between the continuous-time state observer value and its sampled and held version, which is currently used to implement the control.

To simplify the notation, consider the augmented state $x=\left[\begin{array}{ll}\hat{x}^{\top} & e^{\top}\end{array}\right]_{\top}^{\top} \in \mathbb{R}^{2 n}$ and the available signals $y=\left[\begin{array}{ll}\hat{x}^{\top} & e_{y}^{\top}\end{array}\right]^{\top} \in \mathbb{R}^{n+p}$. Thus the closed loop under consideration reads:

$$
\begin{aligned}
& \dot{x}(t)=A x(t)+B \delta(t), \quad \forall t \in\left[t_{k}, t_{k+1}\right) \\
& y(t)=C x(t)
\end{aligned}
$$

with

$$
\begin{gathered}
A=\left[\begin{array}{cc}
A_{p}+B_{p} K & -L C_{p} \\
0 & A_{p}+L C_{p}
\end{array}\right] ; B=\left[\begin{array}{c}
B_{p} K \\
0
\end{array}\right] \\
C=\left[\begin{array}{cc}
I & 0 \\
0 & C_{p}
\end{array}\right] .
\end{gathered}
$$

\section{Problem formulation}

In order to focus on the event-triggered implementation of the controller (2), we want to propose a new dynamic triggering mechanism to enrich the event generator algorithm managing the controller to decide when the control input has to be updated, based on the available information.

Hence, we want to adapt the event-triggered control strategy proposed in [19] and [9]. By considering that a dynamic event-triggering mechanism is introduced through an internal dynamic variable denoted $\eta \in \mathbb{R}$, the sampling instants are determined from the following logic:

$$
t_{k+1}=\min \left\{t \geq t_{k}+T, \text { s.t. } g(y(t), \delta(t), \eta(t)) \leq 0\right\},
$$

where the function $g: \mathbb{R}^{2 n+p+1} \rightarrow \mathbb{R}$ uses the vector of available information to the controller and $T$ is a positive scalar. By definition (10) guarantees that the next sampling time will occur at least $T$ time units ahead the last one. $T$ can be then seen as a minimal dwell time. That allows to prevent Zeno solutions. Furthermore, for $t \geq t_{k}+T$ the control is not updated before $g(y(t), \delta(t), \eta(t)) \leq 0$.

In the paper, we consider an impulsive dynamic of $\eta$ of the following form:

$$
\left\{\begin{aligned}
\dot{\eta}(t)= & f(y(t), \delta(t), \eta(t)), \\
& \forall t \in\left[t_{k}, t_{k}+T\right) \cup\left(t_{k}+T, t_{k+1}\right), \\
\eta\left(t_{k}+T\right)= & \max \left(0, \eta\left(t_{k}+T^{-}\right)\right),
\end{aligned}\right.
$$

for all $k \in \mathbb{N}$, where $f: \mathbb{R}^{2 n+p+1} \rightarrow \mathbb{R}$ and $\eta\left(t_{k}+T^{-}\right)$ denotes the limit of $\eta(t)$ when $t$ approaches $t_{k}+T$ from below. It is further assumed that the dynamic variable $\eta$ is initialized to a value $\eta\left(t_{0}\right) \geq 0$.

The problem we intend to solve in this note can be summarized as follows:

Problem 1: Given a positive scalar $T$, devise functions $f$, $g$ such that the event-triggering mechanism defined as in (10) and (11), ensures the asymptotic stability of the closed-loop system (8), while reducing the number of events generated. 


\section{EVENT-TRIGGERING MECHANISM}

\section{A. Theoretical conditions}

To solve Problem 1, we need to design $T, f$ and $g$ in order to ensure the asymptotic stability of the sampled-data system (5). Let us first propose a general formulation, inspired from [17], [18], which corresponds to extend Theorem 1 of [19] by taking into account the dynamic part of $\eta$ in the same vein as in [9].

Theorem 1: Given a positive scalar $T$, if there exist a function $V: \mathbb{R}^{2 n} \rightarrow \mathbb{R}$, scalars $\epsilon_{1}>0, \epsilon_{2}>0, \epsilon_{3}>0$ and $1>\epsilon_{4}>0$ such that:

1) $\epsilon_{1}\|x\|^{2} \leq V(x) \leq \epsilon_{2}\|x\|^{2}, \quad \forall x \in \mathbb{R}^{2 n}$

2) $\eta\left(t_{k}\right) \geq 0$ and $\eta(t) \geq 0$,

$\forall t \in\left[t_{k}+T, t_{k+1}\right), \forall k \in \mathbb{N}$

3) $\dot{V}(x(t))+\dot{\eta}(t) \leq-\epsilon_{3}(V(x(t))+\eta(t))$,

$\forall t \in\left[t_{k}, t_{k}+T\right) \cup\left(t_{k}+T, t_{k+1}\right), \forall k \in \mathbb{N}$

4) $V\left(x\left(t_{k}+T\right)\right)-V\left(x\left(t_{k}\right)\right) \leq-\epsilon_{4} V\left(x\left(t_{k}\right)\right), \quad \forall k \in \mathbb{N}$

then, the closed-loop system (8) with the triggering mechanism (10) and (11) is asymptotically stable and the interevent intervals are lower bounded by $T$.

Proof: Let us define the function $\mathcal{W}(x, \eta)=V(x)+\eta$ and let $k \in \mathbb{N}$. The stability analysis is carried out considering the time intervals $\left[t_{k}, t_{k}+T\right)$ and $\left[t_{k}+T, t_{k+1}\right)$.

If $\eta\left(t_{k}+T^{-}\right)<0$, then by (11) we have $\eta\left(t_{k}+T\right)=0$. Therefore we have

$$
\begin{aligned}
\mathcal{W}\left(x\left(t_{k}+T\right), \eta\left(t_{k}+T\right)\right) & =V\left(x\left(t_{k}+T\right)\right) \\
& \leq\left(1-\epsilon_{4}\right) V\left(x\left(t_{k}\right)\right) \\
& \leq\left(1-\epsilon_{4}\right) \mathcal{W}\left(x\left(t_{k}\right), \eta\left(t_{k}\right)\right)
\end{aligned}
$$

where the first and second inequalities come from items 4) and 2 ), respectively. If $\eta\left(t_{k}+T^{-}\right) \geq 0$, then by (11) we have $\eta\left(t_{k}+T\right)=\eta\left(t_{k}+T^{-}\right)$. Therefore, we have

$$
\begin{aligned}
\mathcal{W}\left(x\left(t_{k}+T\right), \eta\left(t_{k}+T\right)\right) & =\mathcal{W}\left(x\left(t_{k}+T\right), \eta\left(t_{k}+T^{-}\right)\right) \\
& \leq e^{-\epsilon_{3} T} \mathcal{W}\left(x\left(t_{k}\right), \eta\left(t_{k}\right)\right)
\end{aligned}
$$

where the inequality comes from item 3).

Item 3) also implies that

$$
\mathcal{W}\left(x\left(t_{k+1}\right), \eta\left(t_{k+1}\right)\right) \leq \mathcal{W}\left(x\left(t_{k}+T\right), \eta\left(t_{k}+T\right)\right),
$$

which together with (12), (13) gives for all $k \in \mathbb{N}$,

$$
\mathcal{W}\left(x\left(t_{k+1}\right), \eta\left(t_{k+1}\right)\right) \leq \rho \mathcal{W}\left(x\left(t_{k}\right), \eta\left(t_{k}\right)\right)
$$

where $\rho=\max \left(1-\epsilon_{4}, e^{-\epsilon_{3} T}\right)<1$. Moreover, from items 1) and 2), we have for all $k \in \mathbb{N}$,

$$
\begin{aligned}
\epsilon_{1}\left\|x\left(t_{k}\right)\right\|^{2}+\left|\eta\left(t_{k}\right)\right| \leq \mathcal{W} & \left(x\left(t_{k}\right), \eta\left(t_{k}\right)\right) \\
& \leq \epsilon_{2}\left\|x\left(t_{k}\right)\right\|^{2}+\left|\eta\left(t_{k}\right)\right| .
\end{aligned}
$$

From (6) and (8), one can easily show that there exists $\alpha>1$ such that

$$
\|x(t)\|^{2} \leq \alpha\left\|x\left(t_{k}\right)\right\|^{2}, \forall t \in\left[t_{k}, t_{k}+T\right), \forall k \in \mathbb{N} .
$$

Then, for all $k \in \mathbb{N}$ and for all $t \in\left[t_{k}+T, t_{k+1}\right)$

$$
\begin{aligned}
\epsilon_{1}\|x(t)\|^{2} \leq V(x(t)) & \leq W(x(t), \eta(t)) \\
& \leq W\left(x\left(t_{k}+T\right), \eta\left(t_{k}+T\right)\right) \\
& \leq W\left(x\left(t_{k}\right), \eta\left(t_{k}\right)\right) \\
& \leq \epsilon_{2}\left\|x\left(t_{k}\right)\right\|^{2}+\left|\eta\left(t_{k}\right)\right|
\end{aligned}
$$

where inequalities come from items 1), 2) and 3), (12) and (13) and finally (15). Hence, letting $\beta=\max \left(\alpha, \frac{\epsilon_{2}}{\epsilon_{1}}\right)$ we get

$\|x(t)\|^{2} \leq \beta\left\|x\left(t_{k}\right)\right\|^{2}+\frac{1}{\epsilon_{1}}\left|\eta\left(t_{k}\right)\right|, \forall t \in\left[t_{k}, t_{k+1}\right), \forall k \in \mathbb{N}$.

Then, asymptotic stability follows from (14), (15) and (17). The lower bound on the inter-event time, as $t_{k+1}-t_{k} \geq T$, is guaranteed by the triggering rule (10).

Remark 2: Theorem 1 generalizes to the dynamic eventtriggering laws Theorem 1 provided in [19], which is dedicated to the case of static event-triggering mechanisms.

\section{B. Numerical design of event-triggering mechanism}

In this section, we present numerical techniques to design functions $f, g$ such that the assumptions of Theorem 1 hold. We focus on functions $f$ and $g$ of the following form:

$$
f(y, \delta, \eta)=\left[\begin{array}{c}
y \\
\delta \\
\sqrt{|\eta|}
\end{array}\right]^{\top} Q\left[\begin{array}{c}
y \\
\delta \\
\sqrt{|\eta|}
\end{array}\right]
$$

where $Q \in \mathbb{R}^{(2 n+p+1) \times(2 n+p+1)}$ is a symmetric matrix, and

$$
g(y, \delta, \eta)=\eta
$$

In order to address Problem 1, we need to design $Q$, and the function $V$ complying with the items of Theorem 1 . The following result states matrix inequality conditions in this direction.

Theorem 2: Given the controller and observer gains $K$ and $L$ and a positive scalar $T$, if there exist symmetric positive definite matrix $P \in \mathbb{R}^{2 n \times 2 n}$, a symmetric matrix $Q \in \mathbb{R}^{(2 n+p+1) \times(2 n+p+1)}, \epsilon_{3}>0,1>\epsilon_{4}>0$ such that

$$
\begin{aligned}
& {\left[\begin{array}{ccc}
H e\{P A\}+C^{\top} Q_{1} C+\epsilon_{3} P & P B+C^{\top} Q_{2} & C^{\top} Q_{3} \\
\star & Q_{4} & Q_{5} \\
\star & \star & Q_{6}+\epsilon_{3}
\end{array}\right] \leq 0} \\
& {\left[\begin{array}{cc}
-P & M(T)^{\top} P \\
\star & -\left(1-\epsilon_{4}\right) P
\end{array}\right] \leq 0}
\end{aligned}
$$

with

$$
\begin{gathered}
Q=\left[\begin{array}{ccc}
Q_{1} & Q_{2} & Q_{3} \\
\star & Q_{4} & Q_{5} \\
\star & \star & Q_{6}
\end{array}\right] \\
M(T)=\exp \left(A_{c} T\right)+\int_{0}^{T} \exp \left(A_{c} s\right) d s B\left[\begin{array}{ll}
I_{n} & 0
\end{array}\right] \\
A_{c}=\left[\begin{array}{cc}
A_{p} & -L C_{p} \\
0 & A_{p}+L C_{p}
\end{array}\right],
\end{gathered}
$$

then assumptions of Theorem 1 hold with $V(x)=x^{\top} P x$ and the asymptotic stability of the closed-loop system (8) is guaranteed. Furthermore, the inter-sampling times are lower bounded by $T$. 
Proof: Consider the function $V(x)=x^{\top} P x$, with $P=P^{\top}>0$. Item 1$)$ of Theorem 1 is satisfied with $\epsilon_{1}=\lambda_{\min }(P)$ and $\epsilon_{2}=\lambda_{\max }(P)$.

By (11), we have $\eta\left(t_{k}+T\right) \geq 0$. From continuity of $\eta$ on $\left[t_{k}+T, t_{k+1}\right],(10)$ and (19) give that $\eta(t) \geq 0$ for all $t \in\left[t_{k}+T, t_{k+1}\right]$. Together with $\eta\left(t_{0}\right) \geq 0$, this guarantees the satisfaction of item 2) of Theorem 1.

By computing the time-derivative of $\mathcal{W}(x, \eta)=V(x)+\eta$, for any $t \in\left[t_{k}, t_{k}+T\right) \cup\left(t_{k}+T, t_{k+1}\right)$, along the closedloop system (8) with the dynamics of $\eta$ defined by (11) one gets:

$$
\begin{aligned}
\dot{\mathcal{W}}(x, \eta) & =\dot{V}(x)+\dot{\eta} \\
& =x^{\top}\left(A^{\top} P+P A\right) x+2 x^{\top} P B \delta+f(y, \delta, \eta)
\end{aligned}
$$

Using the form of $f$ given by (18) and the partition of matrix $Q$ as defined in (22), one can write $\dot{\mathcal{W}}$ in a more compact form as follows:

$$
\dot{\mathcal{W}}=\left[\begin{array}{c}
x \\
\delta \\
\sqrt{|\eta|}
\end{array}\right]^{\top} \Phi\left[\begin{array}{c}
x \\
\delta \\
\sqrt{|\eta|}
\end{array}\right]-\epsilon_{3} x^{T} P x-\epsilon_{3}|\eta|
$$

where $\Phi$ corresponds to the matrix in the left-hand side of inequality (20). Thus, the satisfaction of relation (20) ensures that

$$
\left[\begin{array}{c}
x \\
\delta \\
\sqrt{|\eta|}
\end{array}\right]^{\top} \Phi\left[\begin{array}{c}
x \\
\delta \\
\sqrt{|\eta|}
\end{array}\right] \leq 0
$$

That means that

$$
\begin{aligned}
\dot{\mathcal{W}} & \leq-\epsilon_{3} x^{T} P x-\epsilon_{3}|\eta| \\
& \leq-\epsilon_{3}\left(x^{\top} P x+\eta\right)
\end{aligned}
$$

and therefore item 3) in Theorem 1 is satisfied.

In order to prove that item 4) of Theorem 1 holds, one solves (8) over the interval $\left[t_{k}, t_{k}+T\right]$ and it follows:

$$
x\left(t_{k}+T\right)=M(T) x\left(t_{k}\right)
$$

with $M(T)$ defined in (22). Hence, one gets

$$
\begin{gathered}
V\left(x\left(t_{k}+T\right)\right)-V\left(x\left(t_{k}\right)\right) \\
=x\left(t_{k}\right)^{\top}\left(M(T)^{\top} P M(T)-P\right) x\left(t_{k}\right),
\end{gathered}
$$

If relation (21) holds, then $V\left(x\left(t_{k}+T\right)\right)-V\left(x\left(t_{k}\right)\right)=$ $x\left(t_{k}\right)^{\top}\left(M(T)^{\top} P M(T)-P\right) x\left(t_{k}\right) \leq-\epsilon_{4} x\left(t_{k}\right)^{\top} P x\left(t_{k}\right)$. That means that item 4$)$ of Theorem 1 holds.

Hence, Theorem 1 applies and the rest of the result follows.

Remark 3: The case of static event-triggering mechanisms similarly to that one studied in [19] corresponds to remove $\eta$ and consider the event-triggering rule (10) with

$$
g(y, \delta, \eta)=g(y, \delta)=\left[\begin{array}{l}
y \\
\delta
\end{array}\right]^{\top} R\left[\begin{array}{l}
y \\
\delta
\end{array}\right]
$$

with

$$
R=\left[\begin{array}{cc}
R_{\epsilon}^{-1} & 0 \\
\star & -K^{\top} R_{\delta} K
\end{array}\right] \in \mathbb{R}^{(2 n+p) \times(2 n+p)}
$$

Remark 4: The main differences between our Theorems 1 and 2 and Theorem 1 in [6] are: 1) The parameter $T$ directly appears from the sampled system on the interval $\left[t_{k}, t_{k}+T\right]$ and is involved in the decreasing of the Lyapunov function on the interval $\left[t_{k}, t_{k}+T\right]$ without other parameters to fix; 2) In the dynamic of $\eta$ there is a quadratic term in $\sqrt{\eta}$ and matrix $Q$ is only symmetric; 3 ) The considered Lyapunov function is different.

\section{Particular case: state feedback case}

Let us consider the particular case where the state $x_{p}$ is available and therefore no observer is needed. Consider the closed-loop system:

$$
\dot{x}_{p}(t)=\left(A_{p}+B_{p} K\right) x_{p}(t)+B_{p} K \delta(t), \forall t \in\left[t_{k}, t_{k+1}\right)
$$

with $\delta(t)=x_{p}\left(t_{k}\right)-x_{p}(t)$, and the dynamics of $\eta$ is defined by

$$
f\left(x_{p}, \delta, \eta\right)=\left[\begin{array}{c}
x_{p} \\
\delta \\
\sqrt{|\eta|}
\end{array}\right]^{\top} Q\left[\begin{array}{c}
x_{p} \\
\delta \\
\sqrt{|\eta|}
\end{array}\right]
$$

where $Q \in \mathbb{R}^{(2 n+1) \times(2 n+1)}$ is a symmetric matrix. One can handle this case with the framework of Theorem 2 as follows.

Corollary 1: Given the controller gain $K$ and a positive scalar $T$, if there exist symmetric positive definite matrix $P \in$ $\mathbb{R}^{n \times n}$, a symmetric matrix $Q \in \mathbb{R}^{(2 n+1) \times(2 n+1)}$ (partitioned adequately as in (22)), $\epsilon_{3}>0,1>\epsilon_{4}>0$ such that

$$
\begin{gathered}
{\left[\begin{array}{ccc}
H e\left\{P A_{K}\right\}+Q_{1}+\epsilon_{3} P & P B_{p} K+Q_{2} & Q_{3} \\
\star & Q_{4} & Q_{5} \\
\star & \star & Q_{6}+\epsilon_{3}
\end{array}\right] \leq 0} \\
{\left[\begin{array}{cc}
-P & M_{K}(T)^{\top} P \\
\star & -\left(1-\epsilon_{4}\right) P
\end{array}\right] \leq 0}
\end{gathered}
$$

with $A_{K}=A_{p}+B_{p} K$ and

$$
M_{K}(T)=\exp \left(A_{p} T\right)+\int_{0}^{T} \exp \left(A_{p} s\right) d s B_{p} K
$$

then assumptions of Theorem 1 hold with $V\left(x_{p}\right)=x_{p}^{\top} P x_{p}$ and the asymptotic stability of the closed-loop system (24) is guaranteed. Furthermore, the inter-sampling times are lower bounded by $T$.

It is interesting to have a quick focus on the case of state feedback case as studied in [9]. Indeed, the dynamics of $\eta$ used in [9] can be recovered from (25) by considering the following matrix

$$
Q=\left[\begin{array}{ccc}
\sigma Q_{0} & -P B_{p} K & 0 \\
\star & 0 & 0 \\
\star & \star & -\lambda
\end{array}\right] \in \mathbb{R}^{(2 n+1) \times(2 n+1)},
$$

where $Q_{0} \in \mathbb{R}^{n \times n}$ is an arbitrary symmetric positive definite matrix such that $\left(A_{p}+B_{p} K\right)^{\top} P_{0}+P_{0}\left(A_{p}+B_{p} K\right)=-Q_{0}$, with $P_{0}=P_{0}^{\top}>0$ (see relations (15) and (20) in [9]). For this case relation (26) of Corollary 1 simply reads:

$$
\left[\begin{array}{cc}
H e\left\{P A_{K}\right\}+\sigma Q_{0}+\epsilon_{3} P & 0 \\
\star & -\lambda+\epsilon_{3}
\end{array}\right]<0
$$


The interest of the slightly modified version of Corollary 1 (that is replacing (26) by (30)) with respect to results in Section III in [9] resides in the following facts.

- The condition (27) provides a direct way to prevent Zeno solutions thanks to the use of the tunable parameter $T$, avoiding the calculations of Proposition 3.2 in [9];

- We can compute at the same time the adequate parameters $P, \lambda$ and $\sigma$ ( $Q_{0}$ being given as in [9]);

- We can drop the condition $\left(A_{p}+B_{p} K\right)^{\top} P_{0}+P_{0}\left(A_{p}+\right.$ $\left.B_{p} K\right)=-Q_{0}$ since we can replace $\sigma Q_{0}$ by $Q_{1}$ in (30) and therefore search at the same time for $P, Q_{1}$ and $\lambda$.

\section{Optimization issues}

It is interesting to note that the conditions of Theorem 2 are linear matrix inequalities (LMIs) as long as $K, L$ and $T$, $\epsilon_{3}, \epsilon_{4}$ are fixed. Hence, by considering that the gains $K$ and $L$ have been obtained from classical design techniques, and that $T, \epsilon_{3}, \epsilon_{4}$ are a priori fixed to ensure a desired minimal dwell time and convergence rate, conditions (20) and (21) are linear in the decision variables $P$ and $Q_{i}, i=1, \ldots, 6$. Let us emphasize that due to the fact that $A_{p}+B_{p} K$ and $A_{p}+L C_{p}$ are Hurwitz matrices, there always exist positive scalars $T, \epsilon_{3}, \epsilon_{4}$, small enough, such that conditions (20) and (21) are feasible. The objective behind the design of the decision variables is to reduce the amount of control updates. Note that $T$ is a tuning parameter.

Intuitively, a way to reduce the number of control updates is to minimize the conservatism on the estimate of the decay rate given by condition 3 ) in Theorem 1 , or equivalently to maximize the derivative of $\eta$. Numerically, this means that we want the matrix $\Phi$ in the left-hand side of inequality (20) to be as close as possible to zero. The following LMI optimization scheme can then be considered:

$$
\begin{aligned}
& \min \theta \\
& \text { subject to }(20),(21), \Phi \geq-\theta I_{3 n+1} \\
& \text { and } Q_{1}>0, P>I_{2 n}, \operatorname{trace}\left(Q_{1}\right) \leq 1
\end{aligned}
$$

The constraints $P>I_{2 n}$ and $\operatorname{trace}\left(Q_{1}\right) \leq 1$ are added for well conditioning purposes. The addition of the constraint of positive definiteness of $Q_{1}$ allows to enforce the negative definiteness of $H e\{P A\}$, preventing exponentially unstable continuous dynamics and therefore leading to more elegant inter-sample transients.

Moreover, by definition of the event-triggering mechanism, Zeno behavior is avoided because $T$ imposes an explicit minimum inter-event time. The role of $T$ relies on the expected average sampling rate of the event-triggered implementation. An interesting way consists of obtaining the maximal admissible value of $T$ by iteratively increasing $T$ and solving problem (31).

Note that in the case when the used observer is fast, it may be preferable to minimize the conservatism of condition 3) after the observer has converged. In that case, the following
LMI optimization scheme can be considered:

$$
\begin{aligned}
& \min \theta \\
& \text { subject to }(20),(21), D^{\top} \Phi D \geq-\theta I_{3 n+1} \\
& \text { and } Q_{1}>0, P>I_{2 n}, \operatorname{trace}\left(Q_{1}\right) \leq 1
\end{aligned}
$$

where

$$
D=\operatorname{diag}\left(\left[\begin{array}{cc}
I_{n} & 0 \\
0 & 0
\end{array}\right] ; I_{n} ; 0\right) \in \mathbb{R}^{3 n+1}
$$

Remark 5: By considering the state feedback case, and more especially the case treated in [9] and commented in the previous Section III-C, the problem of optimization (31) can be written as follows:

$$
\begin{aligned}
& \min \theta \\
& \text { subject to }(26),(27), \Psi \geq-\theta I_{2 n+1} \\
& \text { and } Q_{1}>0, P>I_{n}, \operatorname{trace}\left(Q_{1}\right) \leq 1
\end{aligned}
$$

where $\Psi$ is the matrix of the left-hand side of inequality (26). In this case one can also obtain the maximal admissible value of $T$ by iteratively increasing $T$ and solving problem (33).

\section{ILLUSTRATIVE EXAMPLES}

In this section, we illustrate the proposed approach through two examples borrowed from [19] and from [9], respectively.

Example 1: By considering the example studied in [19] in which we forget the tracking problem, the system data is defined by:

$$
\begin{aligned}
& A_{p}=\left[\begin{array}{ll}
0 & 1 \\
4 & 0
\end{array}\right], B_{p}=\left[\begin{array}{l}
0 \\
1
\end{array}\right], C_{p}=\left[\begin{array}{ll}
1 & 0
\end{array}\right], \\
& L=\left[\begin{array}{c}
-42 \\
-444
\end{array}\right], K=\left[\begin{array}{ll}
-11.4222 & -6.9135
\end{array}\right] .
\end{aligned}
$$

Since the observer time scale is an order of magnitude faster than the controller time scale, we synthesize a dynamic event-triggering mechanism by solving problem (32) with $T=0.05, \epsilon_{3}=0.1, \epsilon_{4}=1-e^{-\epsilon_{3} T}$. The corresponding closed-loop behavior of the system is shown on Figure 1. One can see that the rate of control updates is high at the beginning of the execution, and tends to decrease after the observer has converged. This is the expected behavior when solving problem (32). If we had solved (31) instead, the contrary behavior would have been observed: large intersampling times at the beginning, which tend to destabilize the system and smaller inter-sampling time after the observer has converged. Of course, such a behavior is not desirable.

Example 2: Consider the example studied in [9] for which we address the state feedback case. The system data is given by:

$$
A_{p}=\left[\begin{array}{cc}
0 & 1 \\
-2 & 3
\end{array}\right], B_{p}=\left[\begin{array}{l}
0 \\
1
\end{array}\right], K=\left[\begin{array}{ll}
1 & -4
\end{array}\right] .
$$

We synthesize a dynamic event-triggering mechanism by solving problem (33) with $T=0.05, \epsilon_{3}=0.99, \epsilon_{4}=1-$ $e^{-\epsilon_{3} T}$. The corresponding closed-loop behavior of the system is shown on Figure 2. Interestingly, the synthesized matrix $Q$ has the particular structure shown in (29). It is also noticeable that the average inter-sampling time is similar to the maximal average inter-sampling time reported in [9]. 

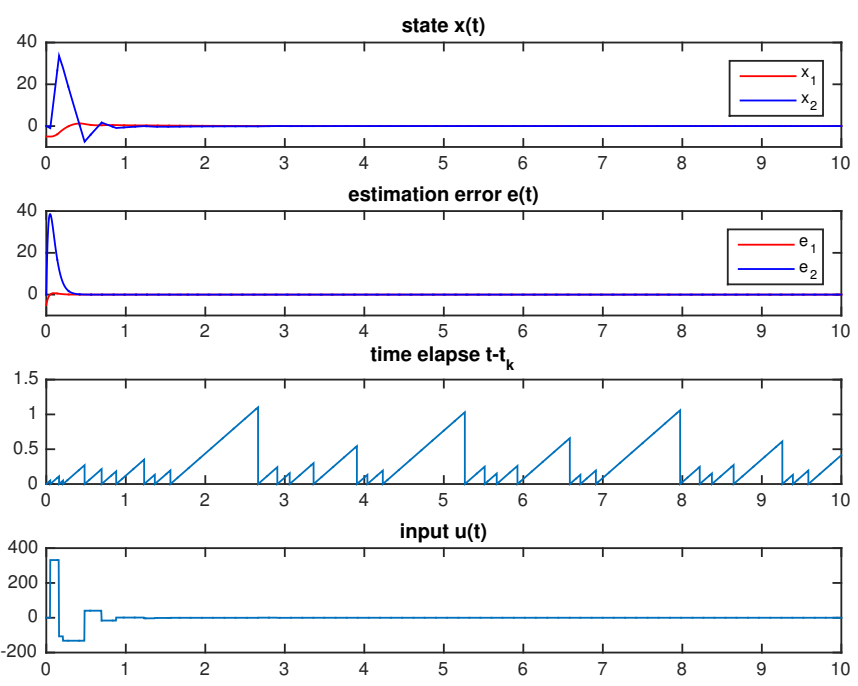

Fig. 1: Closed-loop behavior for system (34), with dynamic event-triggering mechanism synthesized using (32).
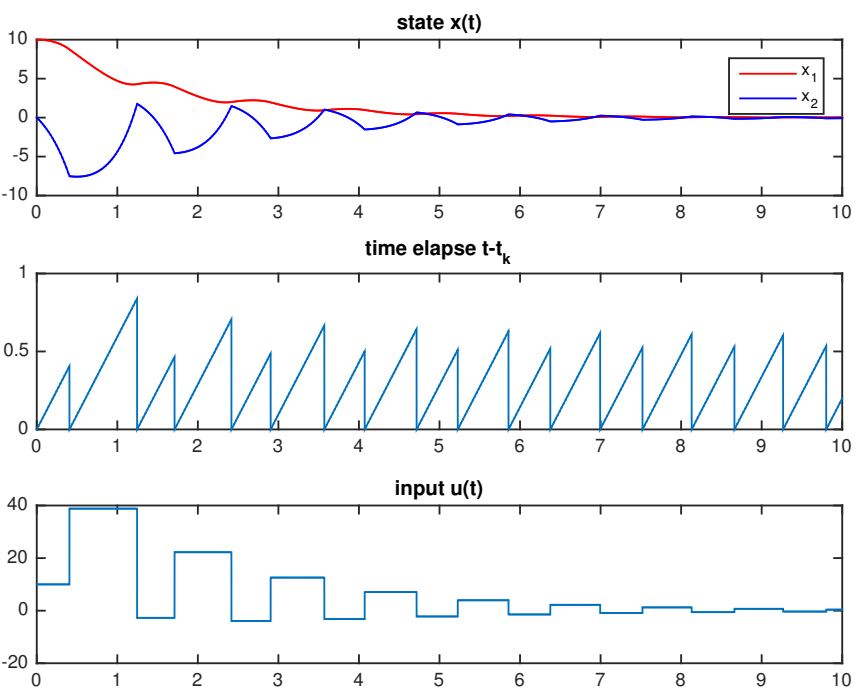

Fig. 2: Closed-loop behavior for system (35), with dynamic event-triggering mechanism synthesized using (33).

\section{Conclusion}

This paper tackled to devise a new dynamic triggering mechanism based on the available information issued from the plant and the observer-based controller. The proposed strategy ensures that Zeno effect is avoided if the sufficient LMI conditions are attended with a tuning positive parameter $T$. Furthermore, a simple optimization criterion allows to design the parameters of the event-triggered law with the implicit objective of reducing the number of control updates.

These results open the door for future research directions. In particular, the co-design problem, that is the design of both the controller and observer gains together with the parameters of the event-triggering mechanism should be carried out. Furthermore, we envision to study what happens with the dynamic event-triggering mechanism when the control system is affected by uncertainty.

\section{REFERENCES}

[1] M. Abdelrahim, R. Postoyan, J. Daafouz, and D. Nešić. Co-design of output feedback laws and event-triggering conditions for linear systems. In 53rd IEEE Conference on Decision and Control, pages 3560-3565, Los Angeles, USA, 2014.

[2] M. Abdelrahim, R. Postoyan, J. Daafouz, and D. Nešić. Stabilization of nonlinear systems using event-triggered output feedback controllers. IEEE Trans. on Automatic Control, 61(9):2682-2687, 2016.

[3] D. Antunes, W. P. M. H. Heemels, and P. Tabuada. Dynamic programming formulation of periodic event-triggered control: Performance guarantees and co-design. In 51th IEEE Conference on Decision and Control, pages 7212-7217, Maui, USA, 2012.

[4] K.J. Aström. Event based control. In Analysis and design of nonlinear control systems, pages 127-147. Springer-Verlag, Berlin Heidelberg, 2008.

[5] D.P. Borgers, V. S. Dolk, and W. P. M. H. Heemels. Riccati-based design of event-triggered controllers for linear systems with delays Riccati-based design of event-triggered controllers for linear systems with delays. IEEE Trans. on Automatic Control, 63(1):174-188, January 2018.

[6] V. S. Dolk, D. P. Borgers, and W. P. M. H. Heemels. Output-based and decentralized dynamic event-triggered control with guaranteed $\mathcal{L}_{p^{-}}$ gain performance and zeno-freeness. IEEE Transactions on automatic control, 62(1):34-49, January 2017.

[7] V. S. Dolk and W. P. M. H. Heemels. Dynamic event-triggered control under packet losses: The case with acknowledgements. In IEEE International Conference on Event-based Control, Communication, and Signal Processing (EBCCSP), pages 1-7, Krakow, Poland, June 2015.

[8] L. Etienne, S. Di Gennaro, and J.P. Barbot. Event triggered observerbased control for linear systems with time varying uncertainties. In American Control Conference (ACC), pages 1531-1536, Chicago, USA, July 2015 .

[9] A. Girard. Dynamic triggering mechanisms for event-triggered control. IEEE Trans. on Automatic Control, 60(7):1992-1996, May 2015.

[10] W. P. M. H. Heemels, M. C. F. Donkers, and A. R. Teel. Periodic event-triggered control for linear systems. IEEE Trans. on Automatic Control, 58(4):847-861, 2013.

[11] W. P. M. H. Heemels, K.H. Johansson, and P. Tabuada. An introduction to event-triggered and self-triggered control. In 51th IEEE Conference on Decision and Control, pages 3270 - 3285, Maui, Hawaii, December 2012.

[12] C. De Persis and P. Frasca. Robust self-triggered coordination with ternary controllers. IEEE Trans. on Automatic Control, 58(12):30243038,2013

[13] R. Postoyan and A. Girard. Triggering mechanism using freely selected sensors for linear time-invariant systems. In 54th Conference on Decision and Control, pages 4812-4817, Osaka, Japan, December 2015.

[14] R. Postoyan, P. Tabuada, D. Nešić, and A. Anta. A framework for the event-triggered stabilization of nonlinear systems. IEEE Trans. on Automatic Control, 60(4):982-996, April 2015.

[15] A. Seuret, C. Prieur, S. Tarbouriech, and L. Zaccarian. LQ-based event-triggered controller co-design for saturated linear systems. Automatica, 74:47-54, 2016.

[16] P. Tabuada. Event-triggered real-time scheduling of stabilizing control tasks. IEEE Trans. on Automatic Control, 52(9):1680-1685, 2007.

[17] P. Tallapragada and N. Chopra. Event-triggered dynamic output feedback control of LTI systems. In 51th IEEE Conference on Decision and Control, pages 6597-6602, Maui, Hawaii, December 2012.

[18] P. Tallapragada and N. Chopra. On event triggered tracking for nonlinear systems. IEEE Trans. on Automatic Control, 58(9):23432348, September 2013.

[19] S. Tarbouriech, A. Seuret, J.-M. Gomes da Silva Jr., and D. Sbarbaro. Observer-based event-triggered control co-design for linear systems. IET Control Theory \& Applications, 10(18):2466-2473, 2016.

[20] X. Wang and M.-D. Lemmon. Event design in event-triggered feedback control systems. In 47th IEEE Conference on Decision and Control, pages 2105-2110, Cancun, Mexico, 2008.

[21] X. Yi, K. Liu, D. Dimarogonas, and K. H. Johansson. Distributed dynamic event-triggered control for multi-agent systems. In IEEE 56th Conference on Decision and Control, pages 6683-6688, Melbourne, Australia, December 2017. 